\title{
Upper airway obstruction resulting from acute mucosal injury induced by direct ingestion of sodium picosulfate/ magnesium citrate powder
}

\section{Gyeong Bo Kim, Sung Yeon Hwang, Tae Gun Shin, Tae Rim Lee, Won Chul Cha, Min Seob Sim, Ik Joon Jo, Keun Jeong Song, Joong Eui Rhee, Yeon Kwon Jeong}

Department of Emergency Medicine, Samsung Medical Center, Sungkyunkwan University School of Medicine, Seoul, Korea

A 59-year-old man presented to the emergency department with a chief complaint of sore throat after swallowing sodium picosulfate/magnesium citrate powder for bowel preparation, without first dissolving it in water. The initial evaluation showed significant mucosal injury involving the oral cavity, pharynx, and epiglottis. Endotracheal intubation was performed for airway protection in the emergency department, because the mucosal swelling resulted in upper airway compromise. After conservative treatment in the intensive care unit, he underwent tracheostomy because stenosis of the supraglottic and subglottic areas was not relieved. The tracheostomy tube was successfully removed after confirming recovery, and he was discharged 3 weeks after admission.

Keywords Caustics; Cathartics; Colonoscopy; Picosulfate sodium

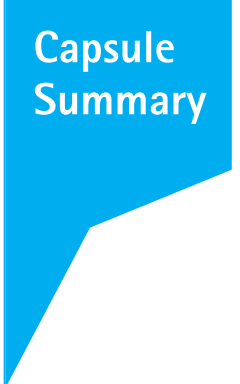

What is already known

Sodium picosulfate/magnesium citrate is one of several colon cleansing agents and its serious side effects are uncommon.

What is new in the current study

Ingestion of undissolved powder of sodium picosulfate/magnesium citrate can cause life-threatening mucosal injury involving the upper airway.
elSSN: 2383-4625

Received: 13 March 2016

Revised: 13 April 2016

Accepted: 14 April 2016

Correspondence to: Tae Gun Shin Department of Emergency Medicine, Samsung Medical Center,

Sungkyunkwan University School of Medicine, 81 Irwon-ro, Gangnam-gu, Seoul 06351, Korea

E-mail: tackles@naver.com

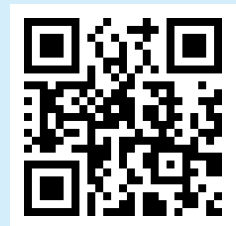

How to cite this article:

Kim GB, Hwang SY, Shin TG, Lee TR, Cha WC, Sim MS, Jo IJ, Song KJ, Rhee JE, Jeong YK. Upper airway obstruction resulting from acute mucosal injury induced by direct ingestion of sodium picosulfate/ magnesium citrate powder. Clin Exp Emerg Med 2016;3(2):109-111.

This is an Open Access article distributed under the terms of the Creative Commons Attribution Non-Commercial License (http:// creativecommons.org/licenses/by-nc/4.0/). 


\section{INTRODUCTION}

Sodium picosulfate/magnesium citrate (SP/MC) powder has been widely used for colon cleansing because it has an acceptable taste and a small volume of liquid is required after dissolving it., ${ }^{1,2}$ The effectiveness, safety, and ease of administration of this orangeflavored powder are well established. ${ }^{3,4}$ Adverse effects are rare in clinical practice, although it may induce mucosal inflammation. ${ }^{5}$ Here, we report the case of a patient who directly ingested SP/ $\mathrm{MC}$ powder without first dissolving it in water, resulting in severe mucosal injury that extended from the oral cavity to the upper esophagus and caused airway compromise.

\section{CASE REPORT}

A 59-year-old man was brought to the emergency department (ED) in an ambulance with a chief complaint of sore throat. He had no significant medical history other than well-controlled hypertension. He was scheduled to undergo colonoscopy that day, and $2 \mathrm{~h}$ prior, he had unintentionally ingested a pack of undissolved SP/MC powder (Picolight powder; Pharmbio Korea Co., Seoul, Korea) that had been prescribed for bowel evacuation. Upon arrival in the ED, he was hemodynamically stable and did not have respiratory distress, but he complained of progressive hoarseness. The emergency physician noted gross mucosal injury of the oropharynx, and examination using a fiberoptic laryngoscope revealed diffuse mucosal swelling and erosion extending from the oral cavity to the epiglottis, which obstructed the view of the vocal cords (Fig. 1). There were no signs of injury at other sites. Laboratory test results and plain chest radiography findings were normal. Endotracheal intubation was performed because his respiratory status was predicted to deteriorate, although he was well oxygenated and ventilated at that time. He was admitted to the intensive care unit for further management. After admission to the inten-
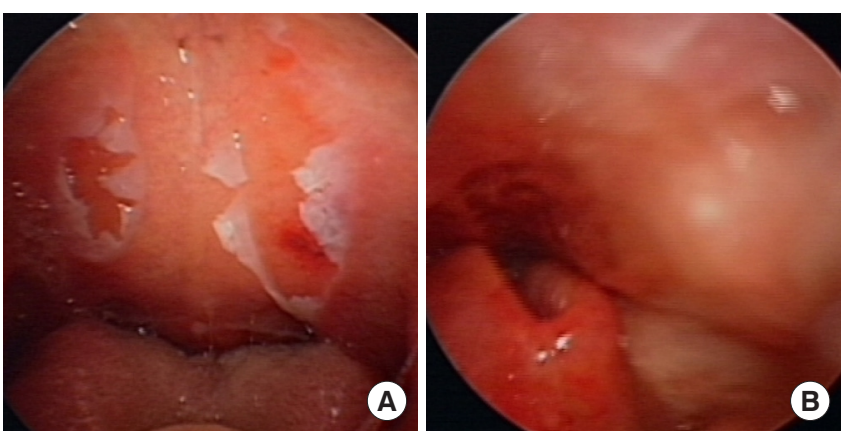

Fig. 1. Fiberoptic laryngoscopic images showing mucosal injury of the oral cavity $(A)$ and supraglottic area obstructing the view of the vocal cords (B). sive care unit, the upper gastrointestinal tract was examined using a flexible endoscope, and mucosal hyperemia, swelling, hemorrhage, and some necrotic changes extending from the oral cavity to the pharynx and epiglottis were observed. Mild hyperemia was noted in the esophagus (Fig. 2); the stomach was not affected.

Supportive therapy was maintained, including intravenous steroid administration to reduce airway swelling, and he was examined regularly to evaluate the airway status and assess the feasibility of extubation. Early tracheostomy was considered, but it was not performed in the first week after admission because he refused the procedure; therefore, we decided to wait for spontaneous recovery. However, on day 12 of hospitalization, fiberoptic examination and computed tomography of the neck revealed persistent stenosis of the supraglottic and subglottic areas (Fig. 3). Therefore, tracheostomy was performed on day 14 of admission, and remnant mucosal damage was still present (Fig. 4). The tra-

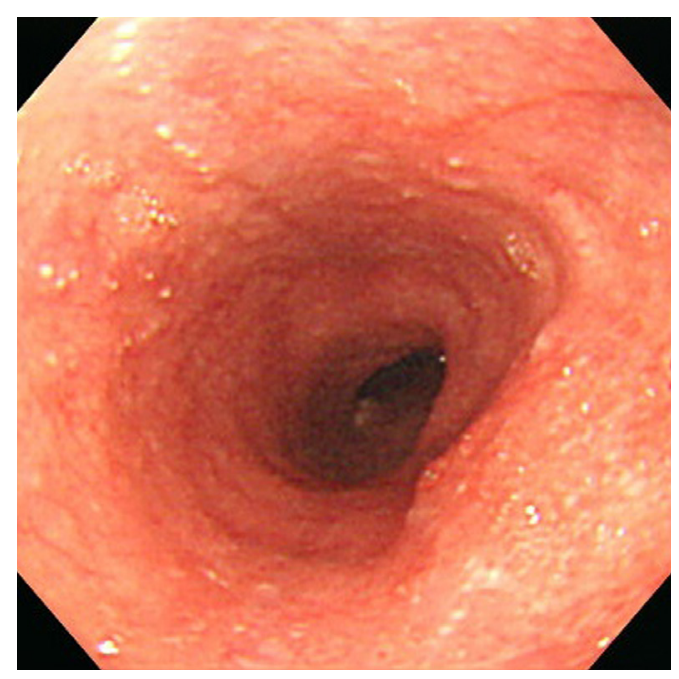

Fig. 2. Esophagogastroduodenoscopy performed at admission showing esophageal hyperemia.

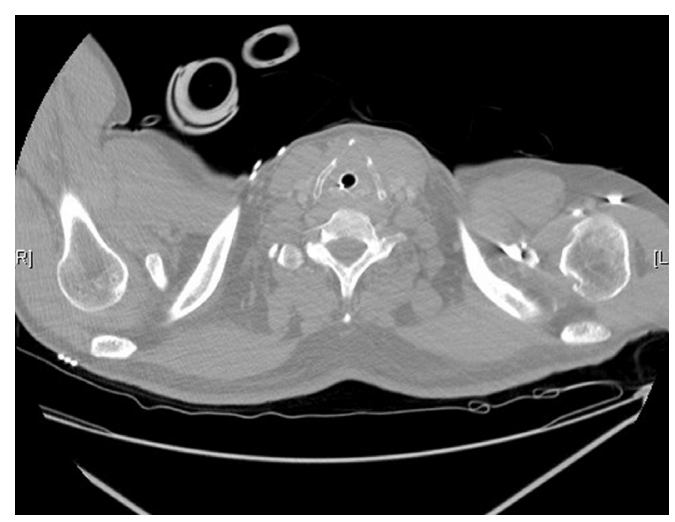

Fig. 3. A computed tomography image showing swelling of the subglottic area surrounding the endotracheal tube. 


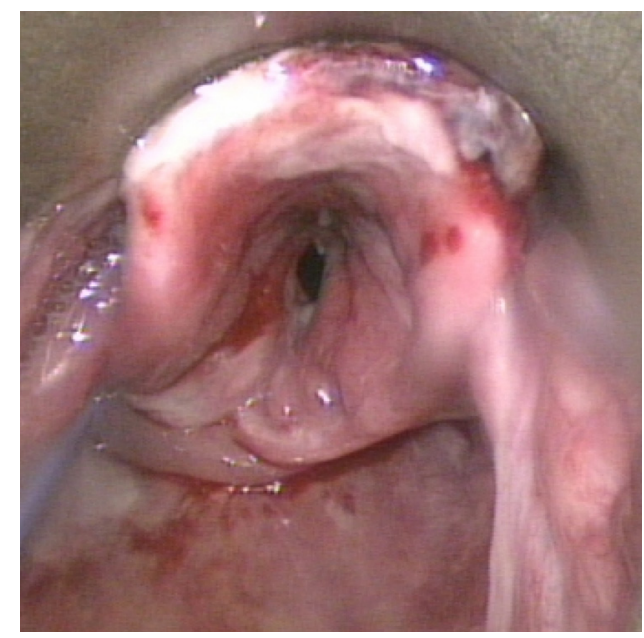

Fig. 4. A fiberoptic laryngoscopic image after tracheostomy.

cheostomy tube was successfully removed after confirming recovery, and the stoma was closed on day 21 of hospitalization. He was discharged the following day. He visited the outpatient clinic for follow-up 1 month after discharge, and no functional or structural sequelae were noted.

\section{DISCUSSION}

SP/MC powder, composed of sodium picosulfate, magnesium oxide, and citric acid, is widely prescribed for bowel preparation. When the powder is dissolved in water, magnesium oxide and citric acid react to form magnesium citrate. ${ }^{6}$ In solution, sodium picosulfate acts as a laxative resulting in augmented peristalsis, and magnesium citrate acts as an osmotic laxative and is not absorbed in the gastrointestinal tract. ${ }^{6,7}$ Side effects of SP/MC are uncommon; however, it has been reported to induce nausea, vomiting, abdominal pain, dehydration, and electrolyte disturbances. ${ }^{5,89}$ The most serious known adverse effect is severe electrolyte imbalance that includes hyponatremia, and rapid worsening of hyponatremia can lead to death. ${ }^{8.9}$

To our knowledge, this is the first report of life-threatening upper airway mucosal damage induced by direct ingestion of SP/ $\mathrm{MC}$ powder. Suh et al. ${ }^{10}$ reported esophageal and gastric mucosal ulcers that resulted from the inadvertent ingestion of SP/MC powder. The mechanism of mucosal injury caused by SP/MC powder is uncertain. However, it may be related to direct thermal injury from heat generated when the powder mixes with a small volume of water or secretions. Citric acid may also cause caustic injury to the mucosa.

Considering that SP/MC powder is commonly used for bowel cleansing, ED physicians and those who prescribe SP/MC powder should be aware that serious mucosal injury can occur if the pow- der is directly ingested without dissolving it in water. To prevent this serious complication, thorough instructions and patient education are important. In particular, health care providers should emphasize that SP/MC powder should be dissolved in an adequate volume of water.

\section{CONFLICT OF INTEREST}

No potential conflict of interest relevant to this article was reported.

\section{REFERENCES}

1. Love J, Bernard EJ, Cockeram A, et al. A multicentre, observational study of sodium picosulfate and magnesium citrate as a precolonoscopy bowel preparation. Can J Gastroenterol 2009; 23:706-10.

2. Song KH, Suh WS, Jeong JS, et al. Effectiveness of sodium picosulfate/magnesium citrate (PICO) for colonoscopy preparation. Ann Coloproctol 2014;30:222-7.

3. Katz PO, Rex DK, Epstein M, et al. A dual-action, low-volume bowel cleanser administered the day before colonoscopy: results from the SEE CLEAR II study. Am J Gastroenterol 2013; 108:401-9.

4. Rex DK, Katz PO, Bertiger G, et al. Split-dose administration of a dual-action, low-volume bowel cleanser for colonoscopy: the SEE CLEAR I study. Gastrointest Endosc 2013;78:132-41.

5. Lawrance IC, Willert RP, Murray K. Bowel cleansing for colonoscopy: prospective randomized assessment of efficacy and of induced mucosal abnormality with three preparation agents. Endoscopy 2011;43:412-8.

6. Hoy SM, Scott $\sqcup$, Wagstaff AJ. Sodium picosulfate/magnesium citrate: a review of its use as a colorectal cleanser. Drugs 2009;69:123-36.

7. Kim DH, Hyun SH, Shim SB, Kobashi K. The role of intestinal bacteria in the transformation of sodium picosulfate. Jpn J Pharmacol 1992;59:1-5.

8. Cho YS, Nam KM, Park JH, Byun SH, Ryu JS, Kim HJ. Acute hyponatremia with seizure and mental change after oral sodium picosulfate/magnesium citrate bowel preparation. Ann Coloproctol 2014;30:290-3.

9. Dillon CE, Laher MS. The rapid development of hyponatraemia and seizures in an elderly patient following sodium picosulfate/magnesium citrate (Picolax). Age Ageing 2009;38:487.

10. Suh JP, Choi YS, Lee SH. Education and imaging. Gastroenterology: acute mucosal injury of esophagus and stomach induced by sodium picosulfate/magnesium citrate for bowel preparation. J Gastroenterol Hepatol 2014;29:1571. 